\title{
Los efectos de una intervención educativa sobre la ingesta de sal para disminuir la sobrehidratación y la hipertensión arterial en pacientes con hemodiálisis
}

\author{
Patricia Arribas Cobo, Anunciacion Fernandez Fuentes, Sonia García Estévez, Ma Ángeles Guimerá Ferré- \\ Samá, Elena Herrera Martín, M ${ }^{a}$ Amor Martínez Aranda
}

\section{Hospital Universitario Infanta Leonor. Madrid}

\section{Introducción y objetivos:}

La patología cardiovascular (CV) representa la principal causa de muerte de pacientes en hemodiálisis (HD), cerca del $50 \%$ y alrededor de un $30 \%$ de las causas de hospitalización (1). La condicionan múltiples factores, tanto clásicos (hipertensión arterial), como específicos de HD (sobrecarga de volumen). La enfermedad cardiovascular altamente prevalente en pacientes en diálisis, está relacionada con exceso de volumen e hipertensión arterial (HTA) (2) Para normalizar el volumen extracelular (VEC) es necesario eliminar agua y sal. El sodio es fundamental (3), la disminución de ingesta de sal, puede disminuir la hipervolemia, la ganancia de peso interdiálisis (GID) e HTA (4). La principal causa del escaso control de PA y peso seco, es la dificultad de restricción de sal en la dieta (5). La HD permite a la enfermera permanecer con el paciente horas, pudiendo realizar educación sanitaria (6) de dieta hiposódica y líquidos, con un proceso sistemático individualizado en el tiempo (7). Es difícil evaluar la ingesta de sal en pacientes en HD y utilizaremos medidas indirectas. La educación sanitaria (8) siempre ha ido dirigida a reducción de ingesta de líquidos, planteamos si es más eficiente, o igual, actuar sobre reducción ingesta de sal. El objetivo del estudio es evaluar efectividad de una intervención educativa sobre la ingesta de sal (9), en términos de disminución de ganancia de peso interdiálisis, disminución de la PA y mejora tolerancia a HD sin deterioro del estado nutricional.

\section{Material y métodos:}

Este estudio consta de dos partes:

- Observacional: caracteriza pacientes en HD con HTA y GID.
- Prospectivo en reducción de sal durante 4 semanas.

Pacientes mayores en HD, 5 meses antes del estudio, (GID) $\geq 2 \mathrm{Kg}$ y/o TA $\geq 140 / 90$ previa a la HD, en más del $30 \%$ de las sesiones, que acepten participación.

La variable primaria disminución GID $\geq 15 \%$, y como secundaria disminución de TA $\geq 2,2 \mathrm{mmHg}$.

Otras variables: parámetros analíticos e interdiálisis, bioimpedancia (10), incidencias intradiálisis.

La encuesta alimentaria se evalúa con programa DIAL.net.

Datos demográficos, clínicos y de diálisis.

La intervención educativa consistió en:

- Formación de formadores, de pacientes sobre su dieta y cuestionario de adherencia.

- Sin cambios en pauta diálisis.

- Se realizó análisis estadístico con programa estadístico SPSS 15.0.

\section{Resultados}

- Se realiza educación de ingesta de sal en 19 pacientes, 12 hombres y 7 mujeres, edad media 65,74 años, peso medio 79,02 y talla 162,76 .

- $31,6 \%$ diabéticos insulinodependientes.

- El 78,9\% tratamiento HD 0N-LINE.

- Todos dializadores alta permeabilidad. 
Resultado de la encuesta dietética es:

- La ingesta media diaria de $6 \mathrm{gr}$ sal, líquidos $1,430 \mathrm{cc}$ bien correlacionadas (11).

- El exceso de sal por productos alimenticios industriales y pan (12).

- El consumo proteínas elevado e ingesta Hidratos de Carbono pobre.

\section{Conclusiones:}

Con Índice de Confianza del 95\% podemos decir que después de la intervención educativa para la reducción de la sal en los pacientes $\mathrm{HD}$, se produce una disminución de la $\mathrm{GID}>15 \%$, que es significativa. No es significativa para TA, pues el periodo de intervención ha sido sólo un mes.

\section{Referencias Bibliográficas}

1. R. Marín,* M. A. Goicoechea, M. Gorostidi, A. Cases, J. Díez, G. Escolar, F. Fernández-Vega, R. Palomar, E. Rodrigo, I. Martínez, y J. Segura, Guía de la Sociedad Española de Nefrología sobre riñón y enfermedad cardiovascular. Nefrología. 2006;26(19).

2. Villar F, Banegas JR, de Mata J, Rodríguez F. Las enfermedades cardiovasculares y sus factores de riesgo en España: hechos y cifras. INFORME SEA 2007. Madrid: Visto Bueno Equipo Creativo, S.L.; 2007.

3. Kooman JP, van der Sande FM, Leunissen KM. Role of sodium and volume in the pathogenesis of hypertension in dialysis patients. Reflections on pathophysiological mechanisms.Blood Purif. 2004, 22 (1) :55-9.

4. Wizemann V, Wabel P, Chamney P, Zaluska W. The mortality risk of overhydration in haemodialysis patients. Nephrol. Dial. Transplant. (2009) 24(5): 1574-1579.

5. Martín LC ,Franco RJS, Gavras I, Matsubara BB, Garcia S, Caramori JT, Barretti BB, Balbi AL, Barsanti R, Padovani C, Gavras H. Association between hypervolemia and ventricular hypertrophy in hemodialysis patients. Am J Hipertension (2004) 17, 1163-1169.

6. Bross R, Noori N, Kovesdy CP, Murali SB, Benner $D$, Block $G$, et al. Dietary assessment of individuals with chronic kidney disease. Semin Dial. 2010 Aug;23(4):359-64.

7. World Health Organization. 2003. Diet nutrition and the prevention of chronic disease. En technical report series 916. Geneva. p 39-42.

8. INTERSALT Cooperative Research Group. An international cooperative study of electrolyte excretion and blood pressure: results for 24 hours urinary sodium and potassium excretion. $\mathrm{Br}$ Med J 1988:319-28.

9. Lindley JL. Reducing sodium intake in hemodialysis patients.Semin Dial . 2009 May-Jun;22(3):260-3.

10. 10. Wabel $P$, Chamney $P$, Moiss $U$, Jirka T. Importance of whole-body bioimpedance spectroscopy for the management of fluid balance. Blood Purif. 2009;27(1):75-80. Pub 2009 Jan 23.

11. Kugler C, Maeding I, Russell CL. Non-adherence in patients on chronic hemodialysis: an international comparison study. J. Nephrol. 2011 Jun;24(3):366-75.

12. de Arpe Muñoz C. «Estudio sobre los patrones de consumo alimentario en España y su relación con el Índice de Masa Corporal, La Hipercolesterolemia, Hipertensión arterial y Diabetes», 2009. 